\title{
A nationwide survey on clinical practice patterns and bleeding complications of percutaneous native kidney biopsy in Japan
}

\author{
Takehiko Kawaguchi ${ }^{1} \cdot$ Tasuku Nagasawsa $^{2} \cdot$ Kazuhiko Tsuruya $^{3} \cdot$ Kenichiro Miura $^{4} \cdot$ Takayuki Katsuno $^{5}$. \\ Takashi Morikawa $^{6} \cdot$ Eiji Ishikawa $^{7} \cdot$ Masao Ogura $^{8} \cdot$ Hideki Matsumura $^{9} \cdot$ Ryota Kurayama $^{10}$. \\ Shinsuke Matsumoto ${ }^{11}$. Yuhji Marui ${ }^{12}$. Shigeo Hara ${ }^{13}$. Shoichi Maruyama ${ }^{14} \cdot$ Ichiei Narita $^{15} \cdot$ Hirokazu Okada $^{16}$. \\ Yoshifumi Ubara ${ }^{17}$ on behalf of Committee of Practical Guide for Kidney Biopsy 2019
}

Received: 18 January 2020 / Accepted: 1 March 2020 / Published online: 18 March 2020

(c) The Author(s) 2020

\begin{abstract}
Background Practice patterns and bleeding complications of percutaneous native kidney biopsy (PNKB) have not recently been investigated and the Japanese Society of Nephrology performed a nationwide questionnaire survey in 2018.

Methods The survey consisted of nine sections about PNKB: (1) general indications; (2) indications for high-risk patients; (3) informed consent; (4) pre-biopsy evaluation; (5) procedures; (6) sedation; (7) post-biopsy hemostasis, bed rest, and examinations; (8) bleeding complications; and (9) specimen processing. A supplementary survey examined bleeding requiring transcatheter arterial embolization (TAE).

Results Overall, 220 directors of facilities (nephrology facility [NF], 168; pediatric nephrology facility [PF], 52) completed the survey. Indications, procedures, and monitoring protocols varied across facilities. Median lengths of hospital stay were 5 days in NFs and 6 days in PFs. Gauge 14, 16, 18 needles were used in 5\%, 56\%, 33\% in NFs and 0\%, 63\%, 64\% in PFs. Mean limits of needle passes were 5 in NFs and 4 in PFs. The bed rest period was 16-24 h in $60 \%$ of NFs and $65 \%$ of PFs. Based on 17,342 PNKBs, incidence rates of macroscopic hematuria, erythrocyte transfusion, and TAE were 3.1\% (NF, 2.8\%; $\mathrm{PF}, 6.2 \%), 0.7 \%$ (NF, 0.8\%; PF, 0\%), and 0.2\% (NF, 0.2\%; PF, 0.06\%), respectively. Forty-six percent of facilities processed specimens all for light microscopy, immunofluorescence, and electron microscopy, and $21 \%$ processed for light microscopy only. Timing of bleeding requiring TAE varied among PNKB cases.

Conclusion Wide variations in practice patterns of PNKB existed among facilities, while PNKBs were performed as safely as previously reported.
\end{abstract}

Keywords Kidney biopsy $\cdot$ Clinical practice pattern $\cdot$ Bleeding complication $\cdot$ Macroscopic hematuria $\cdot$ Erythrocyte transfusion · Transcatheter arterial embolization

\section{Introduction}

Kidney biopsy (KB) is an essential tool for the diagnosis and treatment of patients with numerous kidney diseases. However, its invasive nature results in bleeding complications, leading to increased patient morbidity, longer hospital stay, and higher medical costs. Advancements have been made in

Electronic supplementary material The online version of this article (https://doi.org/10.1007/s10157-020-01869-w) contains supplementary material, which is available to authorized users.

Takehiko Kawaguchi

kawatake45@gmail.com

Extended author information available on the last page of the article biopsy techniques to improve diagnostic yield and to minimize complications, but there remain no global guidelines available to the nephrology community on indications and procedures of $\mathrm{KB}$ to improve diagnosis and prognosis.

The Japanese Society of Nephrology (JSN) originally published the "Guidebook of the renal biopsy" in 2004 to develop best strategies for $\mathrm{KB}$ and to minimize the risk of complications [1]. Over 10 years have passed and the annual number of percutaneous native kidney biopsies (PNKBs) has recently exceeded $10,000[2,3]$. The techniques and devices for PNKB have evolved to improve safety profiles, while high-risk patients, such as diabetic and elderly kidney disease patients, have been rapidly increasing in number. However, the clinical practice patterns and bleeding 
complications of PNKB have never been comprehensively investigated since the publication of the guidebook.

This study aimed to obtain information on the current clinical practice patterns and bleeding complications of PNKB in Japan. The survey could be helpful in providing a reliable consensus and developing better guidelines for PNKB.

\section{Materials and methods}

\section{Data source}

The JSN designed a nationwide questionnaire survey of medical directors of nephrology and pediatric nephrology in Japanese hospitals in 2017. The questionnaire was developed through an iterative review process with the Committee members of the Practical Guide for Kidney Biopsy 2019 of the JSN, to clarify clinical practice patterns and bleeding complications of KB in Japan.

The survey consisted of 70 questions about PNKB, with the exception of transplanted graft biopsy, in nine sections: (1) general indications; (2) indications for high-risk patients; (3) informed consent; (4) pre-biopsy evaluation; (5) biopsy procedures; (6) sedation; (7) post-biopsy hemostasis, bed rest, and examinations; (8) incidence rate of bleeding complications; and (9) specimen processing. The complete survey is described in the supplementary material (Supplement 1).

The JSN distributed the anonymous online survey to nephology teaching hospitals designated by the JSN in February 2018. Respondents were limited to only one director of each facility. Pediatric nephrologists were not asked questions regarding kidney size, since kidney sizes among children vary by age. Conversely, only pediatric nephrologists were asked questions on sedation, since sedation is not routinely performed during PNKB in adult patients. The incidence rates of bleeding complications were investigated over a 3-year period (from January 2015 to December 2017).

The JSN also conducted an anonymous online survey in September 2018 on serious bleeding cases with transcatheter arterial embolization (TAE) after PNKB, which had emerged during the original survey in February 2018. The supplementary questionnaire survey was designed separately from the original to clarify clinical background of hemorrhage events requiring TAE over a period of 5 years (from January 2013 to December 2017). The complete survey is described in the Supplement 2.

These surveys were approved by the ethics committee of the JSN (approvals \#53 and \#58 in 2018). Multiple approaches to improving participation were implemented, including outreach to the directors through email and verbal contact, reminders through the JSN website, and extending the participation deadline of the surveys.

\section{Statistical analysis}

Standard descriptive statistics were used to describe the practice patterns and incidence rates of complications of PNKB. Percentages, medians (interquartile ranges [IQRs]), and means (standard deviations [SDs]) were reported as appropriate. Data regarding nephrology facilities (NFs) for adult patients and pediatric nephrology facilities (PFs) for child and adolescent patients were analyzed separately, except for the section on specimen processing. In the analyses of incidence rates of bleeding complications, facilities with missing data on minor or major complications were excluded. Only facilities having complete data for both minor and major complications over the 3 years of the survey period were included in the analyses to adequately compare incidence rates of the complications. All statistical analyses were performed using Stata version 14.2 (Stata Corp., College Station, Texas, USA).

\section{Results}

\section{Respondent demographics}

Overall, 224 of 667 directors of Japanese nephrology teaching hospitals responded and of these 220 directors (NF, 168; PF, 52) completed the survey (33\% response rate). The annual number of PNKBs performed varied depending on the facilities (range 0-193) and the number of PNKBs in all facilities totaled 24,238 for the 3-year period (average: 8079 per year).

\section{Section 1: General indications for kidney biopsy}

\section{Isolated hematuria}

Twenty-six percent and $60 \%$ of respondents in NFs and PFs, respectively, did not regard isolated hematuria (IH) as an indication for PNKB. Conversely, patients with IH with macroscopic/gross hematuria, suspected of having IgA nephropathy (IgAN) or hereditary nephritis were considered a specific indication (positive response, 38\%, 67\%, and $62 \%$ of all facilities, respectively). Sixty-two percent of NFs, but only $4 \%$ of PFs, recommended a biopsy with IH in the presence of dysmorphic urinary red blood cells of glomerular origin. 


\section{Isolated proteinuria}

Seventy percent of respondents from NFs and 50\% in PFs did not regard isolated proteinuria (IP) less than $0.5 \mathrm{~g} /$ day as an indication for PNKB, while IP of $0.5 \mathrm{~g} /$ day or more in chronic kidney disease (CKD) stage G1, G2, and G3 was considered an indication (Table 1). In advanced CKD G stages, the percent of positive responses increased according to proteinuria or higher CKD A stage in NFs, but remained unchanged according to proteinuria in PFs. IP with suspected hereditary nephritis was considered a specific indication (79\% of all facilities). Ninety percent of respondents in NFs and 38\% in PFs recommended or experienced a biopsy for IP in the presence of dysproteinemia or tubular proteinuria.

\section{Proteinuria with hematuria}

In contrast to IP, proteinuria less than $0.5 \mathrm{~g} /$ day with hematuria was regarded as an indication for PNKB in over $60 \%$ of respondents in NFs and 50\% in PFs (Table 2). Hematuria and proteinuria of $0.5 \mathrm{~g} /$ day or more in CKD stage $\mathrm{G} 1, \mathrm{G} 2$, and G3 were considered an indication. In advanced CKD G stages, the proportion of positive answers increased according to proteinuria or higher CKD A stage in NFs, but remained unchanged according to proteinuria status in PFs.

\section{Rapidly progressive glomerulonephritis or acute renal failure}

PNKB for rapidly progressive glomerulonephritis (RPGN), such as antineutrophil cytoplasmic antibody (ANCA)-associated nephritis and anti-glomerular basement membrane (GBM) nephritis, and acute renal failure (ARF), including drug-induced nephropathy, were recommended or performed in most facilities (positive response for RPGN: $98 \%$ of NFs and $89 \%$ of PFs; for ARF: $89 \%$ of NFs and $67 \%$ of PFs). However, the indication for PNKB for RPGN or ARF varied according to kidney function. The proportion of positive responses for RPGN or ARF with estimated glomerular filtration rate (eGFR) less than 60,30 , and $15 \mathrm{~mL} / \mathrm{min} / 1.73$ $\mathrm{m}^{2}$ was $89 \%, 79 \%$, and $63 \%$ of NFs, and $71 \%, 50 \%$, and $33 \%$ of PFs, respectively.

\section{Systemic diseases}

High proportions of all respondents gave positive answers for PNKB indications involving urinary abnormalities with systemic diseases, such as systemic lupus erythematosus
Table 1 Indications or experiences of kidney biopsy for patients with isolated proteinuria

\begin{tabular}{|c|c|c|c|c|c|c|c|c|}
\hline \multirow{3}{*}{ CKD G stage } & \multicolumn{8}{|c|}{ Urinary protein } \\
\hline & \multicolumn{2}{|c|}{$\geq 0.15$ g/day $(\%)$} & \multicolumn{2}{|c|}{$\geq 0.5$ g/day $(\%)$} & \multicolumn{2}{|c|}{$\geq 1$ g/day $(\%)$} & \multicolumn{2}{|c|}{$\begin{array}{l}\geq 3.5 \mathrm{~g} / \text { day } \\
(\%)\end{array}$} \\
\hline & NF & PF & NF & $\mathrm{PF}$ & $\mathrm{NF}$ & $\mathrm{PF}$ & $\mathrm{NF}$ & $\mathrm{PF}$ \\
\hline G1 (eGFR > 90) & 24 & 29 & 83 & 88 & 98 & 94 & 98 & 94 \\
\hline G2 (eGFR 60-90) & 29 & 44 & 87 & 85 & 98 & 87 & 98 & 87 \\
\hline G3 (eGFR 30-60) & 29 & 52 & 79 & 77 & 96 & 79 & 98 & 81 \\
\hline G4 (eGFR 15-30) & 17 & 31 & 38 & 46 & 52 & 50 & 66 & 52 \\
\hline G5 $(\mathrm{eGFR}<15)$ & 8 & 19 & 11 & 27 & 18 & 31 & 31 & 31 \\
\hline
\end{tabular}

$N F$ nephrology facilities for adult patients, $P F$ pediatric nephrology facilities for child and adolescent patients

\begin{tabular}{|c|c|c|c|c|c|c|c|c|}
\hline \multirow{3}{*}{ CKD G stage } & \multicolumn{8}{|c|}{ Urinary protein } \\
\hline & \multicolumn{2}{|c|}{$\geq 0.15$ g/day $(\%)$} & \multicolumn{2}{|c|}{$\geq 0.5 \mathrm{~g} /$ day $(\%)$} & \multicolumn{2}{|c|}{$\geq 1 \mathrm{~g} /$ day $(\%)$} & \multicolumn{2}{|c|}{$\begin{array}{l}\geq 3.5 \mathrm{~g} / \text { day } \\
(\%)\end{array}$} \\
\hline & $\mathrm{NF}$ & $\mathrm{PF}$ & $\mathrm{NF}$ & $\mathrm{PF}$ & $\mathrm{NF}$ & $\mathrm{PF}$ & $\mathrm{NF}$ & $\mathrm{PF}$ \\
\hline G1 (eGFR > 90) & 64 & 52 & 96 & 100 & 99 & 100 & 99 & 100 \\
\hline G2 (eGFR 60-90) & 67 & 65 & 98 & 92 & 100 & 92 & 100 & 92 \\
\hline G3 (eGFR 30-60) & 61 & 62 & 91 & 79 & 97 & 79 & 98 & 79 \\
\hline G4 (eGFR 15-30) & 32 & 38 & 48 & 48 & 58 & 50 & 68 & 52 \\
\hline G5 $(\mathrm{eGFR}<15)$ & 15 & 25 & 25 & 31 & 30 & 31 & 39 & 33 \\
\hline
\end{tabular}

$N F$ nephrology facilities for adult patients, $P F$ pediatric nephrology facilities for child and adolescent patients
Table 2 Indications or experiences with kidney biopsy for patients with concomitant proteinuria and hematuria 
(SLE, 96\%) and vasculitis (VA, 97\%). Interestingly, SLE and VA with no urinary abnormalities also presented indications for PNKB in a number of facilities (SLE: $45 \%$ of NFs and $81 \%$ of PFs, VA: $52 \%$ of NFs and $29 \%$ of PFs). Other diseases or conditions not associated with urinary abnormalities were also indications for PNKB, including enlarged kidney or kidney dysfunction of unknown cause; IgG4related diseases; hypocomplementemia; hereditary diseases, such as Fabry disease; and positive tubular injury markers, such as $\beta 2$ microglobulin (MG), $\alpha 1 \mathrm{MG}$, and $N$-acetyl- $\beta$-D glucosaminidase.

\section{Diabetic kidney disease}

Diabetes mellitus (DM) with urinary abnormalities was an indication for PNKB in $88 \%$ of respondents in NFs and $11 \%$ in PFs. Surprisingly, only $2 \%$ of NFs, but $38 \%$ of PFs had no experience with PNKB in cases with diabetic kidney disease (DKD). Indications for PNKB of DKD varied according to CKD G stage and proteinuria (Table 3). DKD with hematuria, rapid increase in proteinuria, and absence of retinopathy were also considered an indication for PNKB in NFs $(88 \%$, $88 \%$, and $89 \%$, respectively).

\section{Elderly patients}

The upper age limit for PNKB was 79 years in 20\%, 89 years in $57 \%$, and 90 years or older in $13 \%$ of NFs, while a minority of nephrologists regarded less than 75 years of age as an indication for PNKB (the upper age limit of 64 years in $0 \%$, 69 years in $2 \%$, and 74 years in $6 \%$ of NFs).

\section{Hereditary diseases}

A number of respondents in both NFs and PFs regarded hereditary diseases as an indication for PNKB or experienced a PNKB of hereditary diseases, such as Alport syndrome (positive answer, $74 \%$ ), mitochondrial disease (22\%), nephronophthisis (27\%), and autosomal dominant tubulointerstitial kidney disease (12\%).

\section{Section 2: Indications for kidney biopsy in high-risk patients}

Table 4 shows the indications or experience with $\mathrm{KB}$ in high-risk patients. High-risk patients were not necessarily contraindicated for PNKB in many facilities of nephrology experts. The survey also indicated that the median of the upper limit of body mass index (BMI) for KB was 35 (IQR 30-35). Additionally, 33\% of respondents had experienced a case diagnosed with malignancy by KB in NFs, while there was no experience in PFs.

\section{Section 3: Informed consent for kidney biopsy}

PNKB was commonly performed in inpatient settings and only one NF $(0.5 \%$ overall $)$ performed PNKB in the outpatient setting. Informed consent (IC) was obtained for PNKB in an outpatient consultation room ( $65 \%$ of all facilities), at bedside after hospitalization $(55 \%)$, or in a patient interview room (31\%). IC for blood transfusion was routinely obtained in advance of PNKB only in $26 \%$ of NFs and $35 \%$ of PFs. Clinical paths were utilized for hospitalization of PNKB in $88 \%$ of NFs and $77 \%$ of PFs. The lengths of hospital stay were 4-6 days in 79\% of NFs and 6 days or more in $71 \%$ of PFs. PNKB was performed the next day after admission in $77 \%$ of all facilities.

\section{Section 4: Pre-biopsy evaluations}

Pre-biopsy evaluations included assessments of bleeding diathesis, kidney function, kidney size, blood pressure, and anemia. The cutoff values of contraindications for PNKB are described in Table 5. Prothrombin timeinternational normalized ratio (PT-INR), activated partial thromboplastin time (APTT), bleeding time (BT), and platelet count were screened for indications for PNKB in
Table 3 Indications or experiences of kidney biopsy for patients with diabetic kidney disease

\begin{tabular}{|c|c|c|c|c|c|c|c|c|c|c|}
\hline \multirow{3}{*}{ CKD G stage } & \multicolumn{10}{|c|}{ Urinary protein } \\
\hline & \multicolumn{2}{|c|}{$\begin{array}{l}<0.15 \text { g/day } \\
(\%)\end{array}$} & \multicolumn{2}{|c|}{$\begin{array}{l}\geq 0.15 \mathrm{~g} / \text { day } \\
(\%)\end{array}$} & \multicolumn{2}{|c|}{$\geq 0.5 \mathrm{~g} /$ day $(\%)$} & \multicolumn{2}{|c|}{$\geq 1$ g/day $(\%)$} & \multicolumn{2}{|c|}{$\begin{array}{l}\geq 3.5 \mathrm{~g} / \text { day } \\
(\%)\end{array}$} \\
\hline & NF & $\mathrm{PF}$ & NF & $\mathrm{PF}$ & $\mathrm{NF}$ & $\mathrm{PF}$ & $\mathrm{NF}$ & $\mathrm{PF}$ & $\mathrm{NF}$ & $\mathrm{PF}$ \\
\hline G1 (eGFR > 90) & 10 & 2 & 19 & 10 & 42 & 13 & 64 & 17 & 77 & 63 \\
\hline G2 (eGFR 60-90) & 12 & 4 & 21 & 6 & 45 & 10 & 68 & 12 & 82 & 12 \\
\hline G3 (eGFR 30-60) & 14 & 4 & 22 & 8 & 43 & 10 & 64 & 12 & 75 & 12 \\
\hline G4 (eGFR 15-30) & 8 & 4 & 11 & 6 & 23 & 6 & 30 & 6 & 36 & 6 \\
\hline G5 $($ eGFR < 15) & 4 & 2 & 4 & 2 & 7 & 2 & 10 & 2 & 14 & 2 \\
\hline
\end{tabular}

$N F$ nephrology facilities for adult patients, $P F$ pediatric nephrology facilities for child and adolescent patients 
Table 4 Indications or experiences of kidney biopsy for patients at high risk

\begin{tabular}{|c|c|c|c|c|c|c|c|c|}
\hline \multirow{3}{*}{ Risks } & \multicolumn{8}{|c|}{ Kidney biopsy } \\
\hline & \multicolumn{2}{|c|}{$\begin{array}{l}\text { Echo- } \\
\text { guided } \\
(\%)\end{array}$} & \multicolumn{2}{|c|}{$\begin{array}{l}\text { Open } \\
(\%)\end{array}$} & \multicolumn{2}{|c|}{$\begin{array}{l}\text { Laparo- } \\
\text { scopic } \\
(\%)\end{array}$} & \multicolumn{2}{|c|}{$\begin{array}{l}\text { Total } \\
(\%)\end{array}$} \\
\hline & NF & $\mathrm{PF}$ & $\mathrm{NF}$ & $\mathrm{PF}$ & NF & $\mathrm{PF}$ & $\mathrm{NF}$ & $\mathrm{PF}$ \\
\hline Unilateral kidney or unilateral atrophic or hypoplastic kidney & 15 & 12 & 20 & 21 & 7 & 0 & 42 & 33 \\
\hline Bilateral atrophic or hypoplastic kidney & 18 & 14 & 3 & 8 & 4 & 0 & 25 & 22 \\
\hline Horseshoe kidney & 7 & 8 & 5 & 2 & 1 & 0 & 13 & 10 \\
\hline $\begin{array}{l}\text { Cystic kidney disease, including ADPKD, ARPKD, nephronophthi- } \\
\text { sis, and ADTKD }\end{array}$ & 11 & 31 & 2 & 4 & 0 & 0 & 13 & 35 \\
\hline Hydronephrosis, including retroperitoneal fibrosis and lupus cystitis & 12 & 21 & 2 & 2 & 1 & 0 & 15 & 24 \\
\hline Malignant hypertension, including scleroderma crisis & 52 & 0 & 1 & 2 & 1 & 0 & 54 & 2 \\
\hline Platelet count less than 50,000, including APS, HUS/TTP, TAFRO & 23 & 6 & 3 & 8 & 1 & 2 & 27 & 16 \\
\hline Pregnancy & 10 & 0 & 1 & 0 & 0 & 0 & 11 & 0 \\
\hline Severe obesity & 49 & 19 & 2 & 2 & 2 & 0 & 53 & 21 \\
\hline
\end{tabular}

$N F$ nephrology facilities for adult patients, $P F$ pediatric nephrology facilities for child and adolescent patients, $A D P K D$ autosomal dominant polycystic kidney disease, ARPKD autosomal recessive polycystic kidney disease, $A D T K D$ autosomal dominant tubulointerstitial kidney disease, APS antiphospholipid syndrome, HUS hemolytic uremic syndrome, TTP thrombotic thrombocytopenic purpura, TAFRO thrombocytopenia, anasarca, myelofibrosis, renal dysfunction, and organomegaly

\begin{tabular}{|c|c|c|}
\hline & NF median (IQR), mean (SD) & PF median (IQR), mean (SD) \\
\hline \multicolumn{3}{|l|}{ Bleeding diathesis } \\
\hline PT-INR & $1.5(1.5-1.6), 1.6(0.3)$ & $1.5(1.5-2.0), 1.6(0.4)$ \\
\hline $\operatorname{APTT}(\mathrm{s})$ & $45(40-50), 47(11)$ & $50(45-50), 52(16)$ \\
\hline Bleeding time (min) & $5.0(5.0-5.0), 4.8(1.6)$ & $5(5-5), 4.8(0.6)$ \\
\hline Platelet count, $\times 10^{4} / \mathrm{mm}^{3}$ & $5.0(5.0-10.0), 6.6(2.5)$ & $9.0(5.0-10.0), 7.5(3.2)$ \\
\hline \multicolumn{3}{|l|}{ Kidney function } \\
\hline Serum creatinine, $\mathrm{mg} / \mathrm{dl}$ & $3.0(2.0-3.0), 3.2(1.5)$ & $3.3(3.0-4.0), 4.0(2.3)$ \\
\hline $\mathrm{eGFR}, \mathrm{ml} / \mathrm{min} / 1.73 \mathrm{~m}^{2}$ & $30(15-30), 25(9.2)$ & $30(20-30), 29(12)$ \\
\hline \multicolumn{3}{|l|}{ Kidney size ${ }^{a}$} \\
\hline Major axis, mm & $80(80-90), 83(68)$ & - \\
\hline Cortical thickness, mm & $9(5-10), 8.8(4.5)$ & - \\
\hline \multicolumn{3}{|l|}{ Blood pressure } \\
\hline Systolic blood pressure, $\mathrm{mmHg}$ & 180 (160-180), $175(18)$ & 150 (140-155), $153(20)$ \\
\hline Diastolic blood pressure, $\mathrm{mmHg}$ & $100(100-110), 106(9)$ & $100(90-100), 96(7)$ \\
\hline \multicolumn{3}{|l|}{ Anemia } \\
\hline Hemoglobin, g/dL & $8.0(7.0-9.0), 8.0(1.1)$ & $7.0(6.0-8.0), 7.1(1.2)$ \\
\hline
\end{tabular}

$N F$, nephrology facilities for adult patients, $P F$ pediatric nephrology facilities for child and adolescent patients


depending on age
$83 \%, 63 \%, 40 \%$, and $98 \%$ of NFs, and $81 \%, 77 \%, 31 \%$, and $100 \%$ of PFs. Platelet transfusions were performed when platelet counts were below the cutoff value in $51 \%$ of NFs and $57 \%$ of PFs. Serum creatinine and eGFR were assessed for indication for PNKB in $57 \%$ and $51 \%$ of NFs, and in $46 \%$ and $60 \%$ of PFs, respectively. The cutoff values of contraindications for PNKB varied enormously. Major axis and cortical thickness of the kidneys were evaluated to determine indication in $73 \%$ and $48 \%$ of NFs. Systolic and diastolic blood pressure (BP) were assessed for biopsy indication in $76 \%$ and $39 \%$ of NFs, and in $42 \%$ and $29 \%$ of PFs, respectively. Hemoglobin level was screened for indication for PNKB in $82 \%$ of NFs and $62 \%$ of PFs. 
Erythrocyte transfusion (ET) was performed below the cutoff value in $63 \%$ of NFs and $33 \%$ of PFs.

\section{Section 5: Procedures of kidney biopsy}

\section{Sampling of specimens}

KB was usually performed percutaneously under ultrasonic guidance with local anesthesia in all facilities, but open biopsy (17\% of NFs and 56\% of PFs) and laparoscopic biopsy ( $5 \%$ of NFs and $0 \%$ of PFs) were performed for highrisk patients. Automatic biopsy needles (biopsy gun) were utilized for KB in $98 \%$ of NFs and $96 \%$ of PFs, while TruCut needles and Silverman needles were used in only a few facilities $(0.9 \%$ and $1.4 \%$ of all facilities). Gauge 14,16 , and 18 needles were usually used for KB in $5 \%, 56 \%$, and $33 \%$ of NFs and in $0 \%, 63 \%$, and $37 \%$ of PFs, respectively. The maximum number of sampled specimens for KB varied across facilities. Two specimens were sampled in $17 \%$ of NFs and $56 \%$ of PFs and three specimens in $38 \%$ of NFs and $23 \%$ of PFs. There was also variation in the limit of needle passes for sampling specimens. Three, 4 , and 5 passes at a maximum were allowed in $11 \%, 15 \%$, and $26 \%$ of NFs, and $17 \%, 25 \%$, and $17 \%$ of PFs, respectively. Thirty-three percent of NFs and 27\% of PFs had no limit of needle passes for sampling specimens.

\section{Maximal barrier precautions}

Maximal barrier precautions for $\mathrm{KB}$ included the use of a cap (positive response, $85 \%$ of NFs and $79 \%$ of PFs), a mask (97\% of all), sterile body gown (68\% of PFs and $54 \%$ of PFs), sterile gloves (98\% of all), and sterile drape (67\% of NFs and $60 \%$ of PFs). The proportion of facilities using all equipment and instruments described above was $38 \%$ of NFs and $35 \%$ of PFs.

\section{Medications and urethral catheter use}

Antibiotics and atropine were regularly used before PNKB in $62 \%$ and $21 \%$ of NFs, and $58 \%$ and $15 \%$ of PFs. An indwelling urethral catheter was routinely used in $56 \%$ of NFs and $15 \%$ of PFs.

\section{Blood pressure and antihypertensive drugs}

Sixty-five percent of NFs and 39\% of PFs had a target for BP during the PNKB. The target varied among facilities, but the most common target for systolic BP was less than $160 \mathrm{mmHg}$ in NFs (62\%) and less than 130 or $140 \mathrm{mmHg}$ in PFs (53\%). The most common target for diastolic BP was less than $100 \mathrm{mmHg}$ in both in NFs (49\%) and PFs (62\%). For adult patients with high $\mathrm{BP}$ during KB, antihypertensive agents were administered orally in $28 \%$, sublingually in $4 \%$, and intravenously in 50\%, and were not used in $27 \%$ of NFs. For pediatric patients, antihypertensive drugs were administered orally in $12 \%$, sublingually in $6 \%$, intravenously in $25 \%$, and were not used in $61 \%$ of NFs.

\section{Section 6: Sedation}

Intravenous anesthesia (IVA) in a hospital ward was performed during the PNKB in $78 \%$ of PFs and the age of indication for IVA varied vastly across facilities. IVA was indicated for all pediatric cases, or patients aged 15 years or younger, in $42 \%$ of facilities. Hydroxyzine was used for induction in $49 \%$. For intravenous anesthetics, midazolam, pentazocine, ketamine, and thiopental or thiamylal were administered in $59 \%, 56 \%, 49 \%$, and $34 \%$ of PFs, respectively. A wide variation was also found in the age of indication for general anesthesia (GA) in the operating room setting during the PNKB. GA was indicated for patients younger than 3 years in $81 \%$ and was indicated for all pediatric cases, or patients aged 15 years or younger, in $12 \%$ of PFs. Open biopsy was indicated for patients aged less than 1 year in $86 \%$ of PFs.

\section{Section 7: Post-biopsy hemostasis, bed rest, and examination}

\section{Hemostasis}

Manual compression on the biopsy site was performed soon after PNKB in $95 \%$ of NFs and $100 \%$ of PFs. The median manual compression periods were 10 (IQR 10-15) $\mathrm{min}$ in NFs and 15 (IQR 10-15) min in PFs. Sandbags were used for compression on the biopsy site in $62 \%$ of NFs and $63 \%$ of PFs. The median compression periods using sandbags were 4 (IQR 2-6) h in NFs and 6 (IQR 3-12) h in PFs. Abdominal taping or bandage was used over the biopsy sites in $78 \%$ of NFs and $85 \%$ of PFs. The median duration of abdominal taping or bandaging was 16 (IQR 6-20) $\mathrm{h}$ in NFs and 20 (IQR 14-24) $\mathrm{h}$ in PFs. It depended largely on facilities when discontinued anticoagulant or antiplatelet drugs before PNKB could be restarted after biopsy. The mean duration of drug discontinuation was 2 (IQR 1-7) days in NFs and 7 (IQR 2-7) days in PFs. Hemostatic agents were routinely used after PNKB in $66 \%$ of NFs and $60 \%$ of PFs. Carbazochrome sodium sulfonate hydrate and tranexamic acid were regularly used in $96 \%$ and $100 \%$ of NFs and in $83 \%$ and $63 \%$ of PFs, respectively.

\section{Bed rest}

The prescribed period of strict bed rest in a supine position after PNKB varied among facilities. Strict bed rest for 4-8 h 
was prescribed in $44 \%$ of NFs and in $33 \%$ of PFs. Total bed rest for 16-24 h overall was prescribed in $60 \%$ of NFs and in $65 \%$ of PFs. The median period of subsequent exercise restriction was 14 (IQR 7-28) days in NFs and 14 (IQR 14-28) days in PFs.

\section{Medical examinations and interventions}

Blood tests were routinely performed for monitoring patients after the PNKB in $95 \%$ of NFs and in $84 \%$ of PFs. The examinations were conducted the day after the PNKB in $68 \%$ of NFs and $51 \%$ of PFs. Ultrasound examinations were routinely performed to confirm hemostasis after KB in $84 \%$ of NFs and $82 \%$ of PFs and were performed immediately after the KB in $70 \%$ of the NFs and in $60 \%$ of the PFs. When severe bleeding occurred after the PNKB, surgical procedures could be adopted in their own hospitals in $84 \%$ of NFs and $80 \%$ of PFs. Transcatheter arterial embolization could also be adopted in their own hospitals in $66 \%$ of NFs and $39 \%$ of PFs.

\section{Section 8: Bleeding complications}

One hundred and eighty-one of the 220 facilities (138 of 168 NFs and 43 of 52 PFs; $82 \%$ of all facilities) and 17,342 of 24,238 PNKBs $(15,657$ of 21,468 from NFs and 1685 of 2770 from PFs: $72 \%$ of all PNKBs) were included in the analyses of incidence rates of bleeding complications. Tables 6, 7 and 8 show the incidence of minor and major bleeding complications after PNKB procedures in Japanese facilities. A minor bleeding complication was defined as a macroscopic hematuria (MH) with no treatment except for rehydration, and major bleeding complications included need for blood transfusion, surgical hemostasis, TAE, bladder lavage and/or nephrectomy and death from severe bleeding. Based on 17,342 PNKBs performed (15,657 in NFs and 1685 in PFs) over the past 3 years, the overall incidence rates of MH, ET, and TAE were $3.1 \%$ (95\% confidence interval [CI] 2.8-3.4), $0.7 \%$ (95\% CI $0.6-0.8$ ), and $0.2 \%$ (95\% CI $0.1-0.3$ ), respectively (Table 6). The incidence rates of MH, ET, and TAE in NFs were 2.8\% (95\% CI 2.5-3.0), 0.8\% (95\% CI 0.6-0.9), and $0.2 \%$ (95\% CI 0.1-0.3) and in PFs were 6.2\% (95\% CI 5.1-7.5), 0\%, and 0.06\% (95\% CI 0.002-0.3) (Tables 7,
Table 6 Bleeding complications of percutaneous native kidney biopsies in all facilities of nephrology and pediatric nephrology
Table 7 Bleeding complications of percutaneous native kidney biopsies in nephrology facilities for adult patients

\begin{tabular}{lllll}
\hline Year & 2015 & 2016 & 2017 & Total \\
\hline Number of facilities & 147 & 146 & 154 & 447 \\
Number of biopsies & 5548 & 5691 & 6103 & 17,342 \\
Macroscopic hematuria with no treatment (\%) & $160(2.9)$ & $178(3.1)$ & $198(3.3)$ & $536(3.1)$ \\
Bleeding complications with treatment (\%) & $60(1.1)$ & $54(1.0)$ & $64(1.0)$ & $178(1.0)$ \\
Erythrocyte transfusion $^{\mathrm{a}}(\%)$ & $38(0.7)$ & $39(0.7)$ & $44(0.7)$ & $121(0.7)$ \\
Surgical hemostasis $^{\mathrm{a}}(\%)$ & $1(0.02)$ & $0(0)$ & $0(0.0)$ & $1(0.006)$ \\
Transcatheter arterial embolization $^{\mathrm{a}}(\%)$ & $14(0.3)$ & $8(0.1)$ & $10(0.2)$ & $32(0.2)$ \\
Bladder lavage $^{\mathrm{a}}(\%)$ & $23(0.4)$ & $17(0.3)$ & $25(0.4)$ & $65(0.4)$ \\
Nephrectomy $^{\mathrm{a}}(\%)$ & $0(0)$ & $0(0)$ & $0(0)$ & $0(0)$ \\
Death from severe bleeding $^{\mathrm{a}}(\%)$ & $0(0)$ & $0(0)$ & $1(0.02)$ & $1(0.006)$ \\
\hline
\end{tabular}

${ }^{\text {a }}$ Multiple selections allowed in the same biopsy

\begin{tabular}{lllll}
\hline Year & 2015 & 2016 & 2017 & Total \\
\hline Number of facilities & 112 & 114 & 117 & 343 \\
Number of biopsies & 4906 & 5192 & 5559 & 15,657 \\
Macroscopic hematuria with no treatment (\%) & $132(2.7)$ & $136(2.6)$ & $163(2.9)$ & $431(2.8)$ \\
Bleeding complications with treatment (\%) $^{\text {Erythrocyte transfusion }}{ }^{\mathrm{a}}(\%)$ & $58(1.2)$ & $50(1.0)$ & $59(1.1)$ & $167(1.1)$ \\
Surgical hemostasis $^{\mathrm{a}}(\%)$ & $38(0.8)$ & $39(0.8)$ & $44(0.8)$ & $121(0.8)$ \\
Transcatheter arterial embolization $^{\mathrm{a}}(\%)$ & $1(0.02)$ & $0(0)$ & $0(0.0)$ & $1(0.006)$ \\
Bladder lavage $^{\mathrm{a}}(\%)$ & $14(0.3)$ & $8(0.2)$ & $9(0.2)$ & $31(0.2)$ \\
Nephrectomy $^{\mathrm{a}}(\%)$ & $21(0.4)$ & $15(0.3)$ & $20(0.4)$ & $56(0.4)$ \\
Death from severe bleeding $^{\mathrm{a}}(\%)$ & $0(0)$ & $0(0)$ & $0(0)$ & $0(0)$ \\
\hline
\end{tabular}

${ }^{a}$ Multiple selections allowed in the same biopsy 
Table 8 Bleeding complications of percutaneous native kidney biopsies in pediatric nephrology facilities for child and adolescent patients

\begin{tabular}{lllll}
\hline Year & 2015 & 2016 & 2017 & Total \\
\hline Number of facilities & 35 & 32 & 37 & 104 \\
Number of biopsies & 642 & 499 & 544 & 1685 \\
Macroscopic hematuria with no treatment (\%) & $28(4.4)$ & $42(8.4)$ & $35(6.4)$ & $105(6.2)$ \\
Bleeding complications with treatment (\%) & $2(0.3)$ & $4(0.8)$ & $5(0.9)$ & $11(0.7)$ \\
Erythrocyte transfusion $^{\mathrm{a}}(\%)$ & $0(0)$ & $0(0)$ & $0(0)$ & $0(0)$ \\
Surgical hemostasis $^{\mathrm{a}}(\%)$ & $0(0)$ & $0(0)$ & $0(0)$ & $0(0)$ \\
Transcatheter arterial embolization $^{\mathrm{a}}(\%)$ & $0(0)$ & $0(0)$ & $1(0.2)$ & $1(0.06)$ \\
Bladder lavage $^{\mathrm{a}}(\%)$ & $2(0.3)$ & $2(0.4)$ & $5(0.9)$ & $9(0.5)$ \\
Nephrectomy $^{\mathrm{a}}(\%)$ & $0(0)$ & $0(0)$ & $0(0)$ & $0(0)$ \\
Death from severe bleeding $^{\mathrm{a}}(\%)$ & $0(0)$ & $0(0)$ & $0(0)$ & $0(0)$ \\
\hline
\end{tabular}

${ }^{\mathrm{a}}$ Multiple selections allowed in the same biopsy
8). Death was attributed to severe bleeding in only one adult case of all PNKBs performed [0.006\% (95\% CI 0.0001-0.03)].

\section{Section 9: Processing of biopsy specimens}

Biopsy specimens were fixed and processed in each facility's laboratory for light microscopy (LM), immunofluorescence (IF), and electron microscopy (EM), in $98 \%, 78 \%$, and $46 \%$ of facilities. Forty-six percent fixed and processed specimens comprehensively for all LM, IF, and EM, and $21 \%$ only for LM.

Assistant operators (39\%) or biopsy operators (31\%) mainly divided biopsy specimens and put them in the fixatives. The specimens were routinely assessed with a microscope before dividing in $60 \%$ of facilities (34\% with a dissecting microscope and $26 \%$ with a light microscope), but were not assessed in 31\%. Biopsy specimens were kept from drying before fixation in $84 \%$ of facilities. The specimens were wrapped with saline-soaked gauze in 52\% and placed directly in normal saline in $24 \%$ of the facilities. Each facility determined how biopsy specimens were to be divided for LM, IF, and EM. Either end of the specimen core was taken for IF and EM, with the remainder divided for LM in $40 \%$ of facilities, and both ends from all cores were taken for IF and EM, with the remainder divided for LM in $21 \%$. Samples were randomly taken from all cores for IF, EM, and LM in $39 \%$ of facilities.

The fixative used was $10 \%$ formalin in $61 \%$ of facilities, while neutral buffered formalin was used in $22 \%$. Formalin alone was used as a fixative in $68 \%$ of the facilities, while both formalin and another fixative, such as picric acid, were used in $16 \%$. Liquid nitrogen, dry ice, dry ice with acetone, hexane bottle in dry ice with acetone, and a deep freezer were used for freezing specimens for processing in $24 \%$, $23 \%, 17 \%, 12 \%$, and $9 \%$ of biopsy samples, respectively.

\section{Supplementary survey: Clinical background of bleeding events requiring TAEs after a PNKB}

In the supplementary questionnaire survey, 40 patients from $29 \mathrm{NFs}$ were identified who underwent TAEs after PNKB over the previous 5 years. No patients requiring TAEs were reported from PFs.

The median age was 56 (IQR 39-69) years and 53\% of the patients were male. The median BMI was 22 (IQR $19-25)$ and $89 \%$ of the patients carried out normal activities of daily living (ADL) without being bedridden or confined to a wheelchair. Approximately 20\% had DM and 63\% had no high-risk background, such as unilateral/ hypoplastic/atrophic kidney, cystic kidney diseases, malignant hypertension, or pregnancy. The PNKBs were all performed in inpatient settings, where anticoagulant and antiplatelet agents were used at biopsy in $7.5 \%$ and $5.0 \%$ of the cases, respectively. More common among the patients with TAE were chronic glomerulonephritis (31\%) and RPGN (28\%) in the clinical diagnoses and IgAN (18\%) and crescentic glomerulonephritis (15\%) in the histological diagnosis. Laboratory values before the PNKB (median [IQR]) were as follows: eGFR, $35(10-70) \mathrm{mL} / \mathrm{min} / 1.73$ $\mathrm{m}^{2}$; hemoglobin, $11.0(9.6-13.2) \mathrm{g} / \mathrm{dL}$; platelet count, 21.5 $(15.5-29.9) \times 10^{4} / \mu \mathrm{L}$; PT-INR, $1.0(1.0-1.2)$; APTT, 29 (27-33) s; and BT, $2.5(2.0-4.0)$ min. Mean systolic and diastolic BP at the PNKB were 137 (IQR 119-158) and 80 (IQR 72-90) mmHg, respectively. Median kidney size (major axis) was 100 (IQR 96-109) $\mathrm{mm}$.

Table 9 shows the procedure used for the PNKB as well as the timing and type of hemorrhage occurring after the PNKB, for those patients who underwent TAE. The biopsy procedures, such as the number of needle passes for sampling specimens and the period of bed rest, varied among the cases. The PNKBs resulted in peri-nephric or intranephric bleeding in $87 \%$ of the cases, while only macrohematuria without peri-nephric or intra-nephric bleeding occurred in $13 \%$. Bleeding requiring TAE was apparent 
Table 9 Biopsy procedures and timing of bleeding after biopsy for patients undergoing transcatheter arterial embolization (TAE)

Biopsy procedures

Needle gauge

14

15

16

17

Needle passes for sampling specimens

1

2

3

4

$\geq 5$

Use of hemostatic agents

Yes

No

Manual compression on the biopsy site after biopsy

$<15$ min

15-30 $\mathrm{min}$

$\geq 30 \mathrm{~min}$

Strict bed rest in a supine position after biopsy

$<2 \mathrm{~h}$

$2-4 \mathrm{~h}$

$4-8 \mathrm{~h}$

$8-12 \mathrm{~h}$

$\geq 12 \mathrm{~h}$

Total bed rest after biopsy

$<8 \mathrm{~h}$

$8-16 \mathrm{~h}$

$16-24 \mathrm{~h}$

$\geq 24 \mathrm{~h}$

Number of kidney biopsies the operators had experienced $<50$

Before the biopsy for the patient with TAE 50-200

$\geq 200$

8

62

3

27

3

32

35

14

16

57

Timing of bleeding requiring TAE after biopsy

Cumulative timing of bleeding

$<6 \mathrm{~h}$

$<12 \mathrm{~h}$

$<24 \mathrm{~h}$

$<7$ days

$\geq 7$ days

within $6 \mathrm{~h}$ after the PNKB in 53\% and within $24 \mathrm{~h}$ in $65 \%$ of the cases, while it became evident after 1 week or later in $20 \%$.

\section{Discussion}

This nationwide survey describes the clinical practice patterns and bleeding complications of PNKB in Japanese nephrology facilities. This is the first comprehensive report showing the wide variations in clinical practice across facilities and the low rate of bleeding complications observed in Japan when PNKBs are performed using current techniques and devices.

First, the survey extensively investigated indications for PNKB. Previous studies did not recommend performing a biopsy for patients with $\mathrm{IH}$, since many patients with $\mathrm{IH}$ might manifest no abnormality or slight changes in histology, requiring no specific therapy with excellent prognosis $[4,5]$. The present survey confirmed these data, but the presence of macroscopic/gross hematuria or asymptomatic hematuria of suspected glomerular origin appeared to be a specific indication for biopsy. Under these conditions, biopsy results could alter patient management. One of the most frequent causes of IH is IgAN. IgAN patients with permanent $\mathrm{IH}$ may not have a favorable outcome and are at high risk of progression of renal damage [6, 7]. It might be better to diagnose and manage IgAN early to reduce the probability of end-stage kidney disease. The survey also suggested that IP of less than $0.5 \mathrm{~g} /$ day was not an indication for PNKB in most cases because of a better prognosis of mild proteinuria without systemic diseases, such as SLE and VA. Conversely, IP in the presence of dysproteinemia or tubular proteinuria or IP of suspected hereditary nephritis was considered a specific indication for biopsy, which could determine specific treatment policy. Concurrent proteinuria and hematuria encouraged nephrologists to perform PNKBs, because the condition could suggest a progressive glomerular disease and predict a poor renal outcome.

RPGN, ARF, and systematic diseases such as SLE and VA presented good indications for PNKB. Interestingly, systemic diseases without urinary abnormalities also presented an indication for PNKB for many nephrologists, since active pathological lesions are often found in patients with systemic diseases without urinary abnormalities (known as "silent lupus nephritis") [8, 9].

Policies on performing PNKB for patients with DM and elderly patients have been changing. DM is now the leading cause of end-stage kidney disease in the world, and the previously established opinion among nephrologists was that $\mathrm{KB}$ for DKD would provide little diagnostic, prognostic, or therapeutic value [10]. However, DKD has been recognized as a new disease entity and a worldwide public health problem [11], and it was revealed in the survey that DM with urinary abnormalities presented an indication for PNKB in many facilities. A previous study suggested that pathology varied enormously across DKD and also 
included nondiabetic glomerular diseases, such as membranous nephropathy [12]. These pathological findings could be potentially amenable to therapies that might improve kidney outcome [13]. Additionally, CKD is increasingly common in the elderly, and the survey revealed that performing PNKB in very elderly patients was not always a contraindication in many facilities. PNKB in very elderly patients was not associated with any greater risk of complications than that in younger patients [14], and biopsy could also be a valuable diagnostic tool that provides crucial diagnostic and prognostic information, and changes treatment approach in geriatric patients [15].

High-risk patients may be contraindicated for PNKB. Potential contraindications include unilateral kidney, bilateral atrophic or hypoplastic kidney, horseshoe kidney, multiple bilateral cysts, hydronephrosis, malignant hypertension, uncorrectable bleeding diathesis, pregnancy, and severe obesity. In the previous Japanese Guidebook [1], most of these high-risk conditions were regarded as contraindications for PNKB, while alternative biopsy techniques could be considered. Surprisingly, the survey revealed that many nephrologists do not necessarily regard these high-risk patients as contraindicated for PNKB in the current era when the techniques and devices have evolved to improve safety profiles. It would be equally important to consider which patients would not or would benefit from a biopsy. A laparoscopic or open kidney biopsy can be a good alternative in selected circumstances [16].

The survey found common practices before performing PNKB included assessment of bleeding diathesis, kidney function, kidney size, blood pressure, and anemia, but cutoff values for these parameters contraindicating PNKB and transfusion policies varied enormously across facilities. A previous study reported that bleeding complications were associated with APTT, PT-INR, BT, and platelet count [17]. Additional risk factors for bleeding included hypertension, reduced GFR, anemia, older age, and the use of a 14-gauge biopsy needle [18-22]. However, it may be difficult to determine strict cutoff values for these contraindications for PNKB because of the different patient backgrounds among the previous investigations. The cutoff values should be carefully examined based on individual benefit and risk of patients.

PNKB was most commonly performed by nephrologists in inpatient settings, and clinical paths were utilized for hospitalization in many Japanese facilities. IC for PNKB was obtained in outpatient settings or after admission, while IC for blood transfusion was not routinely obtained in advance of biopsy in most facilities. The length of hospital stay was usually 4-6 days in NFs and 6 days or more in PFs. The duration of hospitalization was likely based on recommendations from the previous Japanese guidebook indicating that patients should be discharged 4-7 days after PNKB to allow better monitoring of bleeding complications after hemostasis [1]. Previous studies have reported worldwide variation in PNKB performed as an inpatient or outpatient procedure, and suggested that the outpatient setting would be safe with low complication rates and more cost-effective than inpatient biopsies [23, 24]. Most bleeding complications are recognized within 12-24 h of the biopsy, but complications occurring after $24 \mathrm{~h}$ were not extremely rare $(2-10 \%)$ [19, 25, 26]. Thus, it might be better to consider the observation period after PNKB based on the patient risk of bleeding complications.

PNKB was most commonly performed with automatic biopsy needles under local anesthesia, while IVA or GA was often performed during pediatric PNKB. Gauge 16 needles were used in many facilities, but a wide variation was found among facilities in the maximum number of sampled specimens and needle passes. A previous systematic review suggested that the use of 14-gauge needles was associated with higher transfusion rates compared with 16and 18-gauge needles, and that the number of passes did not affect the rate of complications [20]. Additionally, the diagnostic yield did not seem to differ significantly among 14- and 16-gauge needles, while some studies indicate a lower yield with 18-gauge needles [27-30]. These data suggest that the use of 16-gauge needles might be encouraged in most circumstances.

Concerning the prevention of infections, only a minority of facilities used all the equipment and instruments for maximal barrier precautions, while many facilities routinely administered antibiotics regularly for prevention of infections. Although infections during PNKB rarely occurred using sterile techniques, infectious complications have been described in some case series [31], and preventive measures should be carefully taken.

Policies on hemostasis, bed rest, and examination after PNKB varied enormously among facilities. Manual compression on the biopsy site was performed soon after the PNKB in almost all facilities, while it depended on the facilities whether sandbags and abdominal taping/bandage were used for compression. As manual compression and abdominal bandaging may increase the frequency of reflex syncope during PNKB [32], it may be necessary to consider the potential benefit and risk of these maneuvers. Hemostatic agents were routinely administered after PNKB in many facilities, but desmopressin was not used in any of the facilities due to non-coverage by medical insurance in Japan. There was also a wide variation in the prescribed period of bed rest after the PNKB. A shorter period of strict bed rest might safely reduce discomfort in PNKB [33]. Conversely, indwelling urethral catheters were routinely used in many nephrology facilities (but only in a few pediatric facilities) to ensure bed rest and to monitor bleeding. It depended on facilities when a discontinued 
anticoagulant or antiplatelet drug would be restarted after the PNKB. Not only bleeding risk, but also thrombosis risk, after the PNKB should be considered for patients taking these drugs. Ultrasound examinations, as well as blood tests, were routinely performed to confirm hemostasis after the PNKB in many facilities, although their utility in predicting relevant clinical complications or altering management has not been clearly demonstrated in previous studies [34, 35].

The present survey showed that the incidence rates of MH (3.1\%), ET (0.7\%), TAE ( $0.2 \%)$, and death attributed to biopsy $(0.006 \%)$ were as low as those reported in a previous systematic review in 2013 (MH 3.5\%, ET 0.9\%, TAE 0.6\%, death $0.02 \%$ ) [21] and in a recent Japanese report in 2015 (ET $0.5 \%$, TAE $0.1 \%$, death $0.06 \%$ ) [3]. It would be critically important to show the safety of PNKB in the modern era of increased number of PNKB performed in high-risk patients, such as elderly patients with many comorbidities. Although our study revealed that the rates of bleeding complications were relatively low when native kidney biopsies were performed using current techniques and devices in Japan, it is crucial to identify which factors of PNKB are significantly associated with the bleeding complications. Further investigations are needed to examine the associations between clinically relevant factors and the complications observed following PNKB procedures in Japan.

In Japanese facilities performing KB, biopsy specimens were not necessarily fixed and processed in their own laboratories all for LM, IF, and EM. It largely depended on facilities how the specimens were to be divided, fixed and processed. Specimens for LM, IF, and EM require different processing and fixation methods, and it is important for nephrologists to be competent at specimen division and processing for optimum diagnostic and prognostic yield [36, 37].

The supplementary survey clarified the clinical backgrounds of severe hemorrhage requiring TAE after PNKB. The original survey did not reveal a high incidence rate of TAE, but TAE was undoubtedly a serious bleeding complication following the PNKB. There have been a few case reports describing TAE after PNKB [38-40], but the underlying conditions for TAE requirement after PNKB have never been comprehensively examined to date. This additional survey suggested a wide variety of patient characteristics and biopsy procedures among cases requiring TAE. Hemorrhage leading to TAE was apparent most frequently within $6 \mathrm{~h}$, while the timing of bleeding with TAE varied widely. Surprisingly, hemorrhage resulting in TAE became evident a week or later after PNKB in $20 \%$ of the cases. To the best of our knowledge, this is the first study to report on the timing of bleeding complications associated with TAE requirement. It may be difficult to predict when critical bleeding requiring TAE will be identified, while nearly
$90 \%$ of all minor and major bleeding complications occur within $24 \mathrm{~h}$ after PNKB [20, 25]. This information is crucially important to ensure safety of PNKB. Close patient follow-up is necessary to detect both early and late onset of severe bleeding complications after PNKB.

A strength of our survey is the nationwide investigation providing extensive information on clinical practice patterns and bleeding complications of PNKB in the current era in Japan. The number of PNKBs performed in all the facilities participating in this survey amounted to $24,238(17,342$ alone for analyses of complications) over a 3 -year period and was sufficiently large to describe the current approach of the Japanese nephrology community towards PNKB. Despite being only descriptive analyses, the results of the survey are still impressive and informative. The reported practice patterns and estimates of complications provided helpful information in the decision-making process involved for PNKB procedures.

Despite its strengths, the limitations of our survey should be recognized. First, the study was based on a cross-sectional descriptive design, and the reported practice patterns were mostly determined by personal experience and the confidence of the physician with the procedure and were not necessarily based on clinical evidence. It is underlined that the application of the results from this study to clinical practice for PNKB should be done carefully. Next, this was the online questionnaire survey of facility directors, and the response rate was 33\% (220 of 667 facilities). The survey presented a lower response rate than the previous questionnaire surveys among nephrology directors $(60-80 \%)[1,5$, $41,42]$, which may have led to a potential selection bias. The survey was distributed to Japanese teaching hospitals, to which many patients at high risk might be referred, but facilities with high complication rates of PNKB may less likely have responded to the survey, resulting in an underestimation of complications.

In conclusion, the present survey showed wide variations of clinical practice patterns of PNKB according to facilities, while PNKBs were performed as safely as previously reported in Japan. These findings may help provide a reliable consensus among nephrologists and develop better guidelines for PNKB.

Acknowledgments The study was supported by the Japanese Society of Nephrology. The authors would like to thank the members of the Japanese Society of Nephrology and the survey respondents for their contribution and support to the research.

\section{Compliance with ethical standards}

Conflict of interest All the authors submitted conflict of interest statements based on academic society rules, which are managed by the Clinical and Experimental Nephrology (CEN). No authors have declared any competing interest. 
Human and animal rights (with IRB Approval number) The surveys were approved by the ethical committee of the JSN (Approvals \#53 and \#58 in 2018).

Informed consent This was an anonymous online survey to medical directors of nephology teaching hospitals and informed consent was not obtained from the individual directors. The information on the research and the protocol was disclosed prior to the anonymous online survey through email and the JSN website.

Open Access This article is licensed under a Creative Commons Attribution 4.0 International License, which permits use, sharing, adaptation, distribution and reproduction in any medium or format, as long as you give appropriate credit to the original author(s) and the source, provide a link to the Creative Commons licence, and indicate if changes were made. The images or other third party material in this article are included in the article's Creative Commons licence, unless indicated otherwise in a credit line to the material. If material is not included in the article's Creative Commons licence and your intended use is not permitted by statutory regulation or exceeds the permitted use, you will need to obtain permission directly from the copyright holder. To view a copy of this licence, visit http://creativecommons.org/licenses/by/4.0/.

\section{References}

1. Japanese Society of Nephrology. Guidebook of the renal biopsy. Tokyo: Tokyo-Igakusha; 2004 (in Japanese).

2. Sugiyama H, Sato H, Ueda Y, Yokoyama H. Progressive renal diseases: recent advances in diagnosis and treatments. Topics: 1. Diagnosis; 2. Epidemiology of renal disease: Japan Renal Biopsy Registry and Japan Kidney Disease Registry. Nihon Naika Gakkai Zasshi. 2013;102:1083-91 (in Japanese).

3. Yamamoto $\mathrm{H}$, Hashimoto $\mathrm{H}$, Nakamura $\mathrm{M}$, et al. Relationship between hospital volume and hemorrhagic complication after percutaneous renal biopsy: results from the Japanese diagnosis procedure combination database. Clin Exp Nephrol. 2015;19:271-7.

4. Richards NT, Darby S, Howie AJ, et al. Knowledge of renal histology alters patient management in over $40 \%$ of cases. Nephrol Dial Transpl. 1994;9:1255-9.

5. Fuiano G, Mazza G, Comi N, et al. Current indications for renal biopsy: a questionnaire-based survey. Am J Kidney Dis. 2000;35:448-57.

6. Szeto CC, Lai FM, To KF, et al. The natural history of immunoglobulin a nephropathy among patients with hematuria and minimal proteinuria. Am J Med. 2001;110:434-7.

7. Shen P, He L, Li Y, et al. Natural history and prognostic factors of IgA nephropathy presented with isolated microscopic hematuria in Chinese patients. Nephron Clin Pract. 2007;106:c157-c161161.

8. Ishizaki J, Saito K, Nawata M, et al. Low complements and high titre of anti-Sm antibody as predictors of histopathologically proven silent lupus nephritis without abnormal urinalysis in patients with systemic lupus erythematosus. Rheumatology (Oxford). 2015;54:405-12.

9. Moroni G, Depetri F, Ponticelli C. Lupus nephritis: When and how often to biopsy and what does it mean? J Autoimmun. 2016;74:27-40.

10. Dhaun N, Bellamy CO, Cattran DC, et al. Utility of renal biopsy in the clinical management of renal disease. Kidney Int. 2014;85:1039-48.

11. Hirakawa Y, Tanaka T, Nangaku M. Mechanisms of metabolic memory and renal hypoxia as a therapeutic target in diabetic kidney disease. J Diabetes Investig. 2017;8:261-71.
12. Mazzucco G, Bertani T, Fortunato M, et al. Different patterns of renal damage in type 2 diabetes mellitus: a multicentric study on 393 biopsies. Am J Kidney Dis. 2002;39:713-20.

13. Sharma SG, Bomback AS, Radhakrishnan J, et al. The modern spectrum of renal biopsy findings in patients with diabetes. Clin J Am Soc Nephrol. 2013;8:1718-24.

14. Nair R, Bell JM, Walker PD. Renal biopsy in patients aged 80 years and older. Am J Kidney Dis. 2004;44:618-26.

15. Haas M, Spargo BH, Wit EJ, et al. Etiologies and outcome of acute renal insufficiency in older adults: a renal biopsy study of 259 cases. Am J Kidney Dis. 2000;35:433-47.

16. Hogan JJ, Mocanu M, Berns JS. The Native Kidney Biopsy: Update and evidence for best practice. Clin J Am Soc Nephrol. 2016;1:354-62.

17. Nass K, O'Neill WC. Bedside renal biopsy: ultrasound guidance by the nephrologist. Am J Kidney Dis. 1999;34:955-9.

18. Korbet SM. Percutaneous renal biopsy. Semin Nephrol. 2002;22:254-67.

19. Shidham GB, Siddiqi N, Beres JA, et al. Clinical risk factors associated with bleeding after native kidney biopsy. Nephrology (Carlton). 2005;10:305-10.

20. Whittier WL, Korbet SM. Timing of complications in percutaneous renal biopsy. J Am Soc Nephrol. 2004;15:142-7.

21. Corapi KM, Chen JL, Balk EM, et al. Bleeding complications of native kidney biopsy: a systematic review and meta-analysis. Am J Kidney Dis. 2012;60:62-73.

22. Eiro M, Katoh T, Watanabe T. Risk factors for bleeding complications in percutaneous renal biopsy. Clin Exp Nephrol. 2005;9:40-5.

23. Bairy M, Beleed K, Webb AT, et al. Safety of outpatient kidney biopsy: one center's experience with 178 native kidney biopsies. Am J Kidney Dis. 2008;52:631-2.

24. McMahon GM, McGovern ME, Bijol V, et al. Development of an outpatient native kidney biopsy service in low-risk patients: a multidisciplinary approach. Am J Nephrol. 2012;35:321-6.

25. Simard-Meilleur MC, Troyanov S, Roy L, et al. Risk factors and timing of native kidney biopsy complications. Nephron Extra. 2014;4:42-9.

26. Prasad N, Kumar S, Manjunath R, et al. Real-time ultrasoundguided percutaneous renal biopsy with needle guide by nephrologists decreases post-biopsy complications. Clin Kidney J. 2015;8:151-6.

27. Mai J, Yong J, Dixson H, et al. Is bigger better? A retrospective analysis of native renal biopsies with 16 Gauge versus 18 Gauge automatic needles. Nephrology (Carlton). 2013;18:525-30.

28. Roth R, Parikh S, Makey D, et al. When size matters: diagnostic value of kidney biopsy according to the gauge of the biopsy needle. Am J Nephrol. 2013;37:249-54.

29. Nicholson ML, Wheatley TJ, Doughman TM, et al. A prospective randomized trial of three different sizes of core-cutting needle for renal transplant biopsy. Kidney Int. 2000;58:390-5.

30. Manno C, Strippoli GF, Arnesano L, et al. Predictors of bleeding complications in percutaneous ultrasound-guided renal biopsy. Kidney Int. 2004;66:1570-7.

31. Parrish AE. Complications of percutaneous renal biopsy: a review of 37 years' experience. Clin Nephrol. 1992;38:135-41.

32. Takeuchi Y, Ojima Y, Kagaya S, et al. Manual compression and reflex syncope in native renal biopsy. Clin Exp Nephrol. 2018;22:1100-7.

33. Ishikawa E, Nomura $\mathrm{S}$, Obe $\mathrm{T}$, et al. How long is strict bed rest necessary after renal biopsy? Clin Exp Nephrol. 2009;13:594-7.

34. Waldo B, Korbet SM, Freimanis MG, et al. The value of postbiopsy ultrasound in predicting complications after percutaneous renal biopsy of native kidneys. Nephrol Dial Transpl. 2009;24:2433-9. 
35. Ishikawa E, Nomura S, Hamaguchi T, et al. Ultrasonography as a predictor of overt bleeding after renal biopsy. Clin Exp Nephrol. 2009;13:325-31.

36. Walker PD, Cavallo T, Bonsib SM. Ad Hoc Committee on Renal Biopsy Guidelines of the Renal Pathology Society. Practice guidelines for the renal biopsy. Mod Pathol. 2004;17:1555-633.

37. Amann K, Haas CS. What you should know about the work-up of a renal biopsy. Nephrol Dial Transpl. 2006;21:1157-61.

38. Ito $\mathrm{T}$, Ishikawa $\mathrm{E}$, Ito M. Lumbar artery injury following renal biopsy. Clin Exp Nephrol. 2016;20:145-6.

39. Krejčí K, Černá M, Žamboch K, et al. Late rupture of lumbar artery as an unusual complication after renal biopsy - case report. Urol Int. 2017;98:112-4.
40. Takeuchi Y, Ojima Y, Kagaya S, et al. Unexpected delayed bleeding after native renal biopsy: a case report. CEN Case Rep. 2018;7:9-12.

41. Liebman SE, Moore CA, Monk RD, Rizvi MS. What are we doing? A survey of United States Nephrology Fellowship Program Directors. Clin J Am Soc Nephrol. 2017;12:518-23.

42. Yuan CM, Nee R, Little DJ, et al. Survey of kidney biopsy clinical practice and training in the United States. Clin J Am Soc Nephrol. 2018;13:718-25.

Publisher's Note Springer Nature remains neutral with regard to jurisdictional claims in published maps and institutional affiliations.

\section{Affiliations}

Takehiko Kawaguchi ${ }^{1} \cdot$ Tasuku Nagasawsa $^{2} \cdot$ Kazuhiko Tsuruya $^{3} \cdot$ Kenichiro Miura $^{4} \cdot$ Takayuki Katsuno $^{5}$. Takashi Morikawa $^{6} \cdot$ Eiji Ishikawa $^{7} \cdot$ Masao Ogura $^{8} \cdot$ Hideki Matsumura $^{9} \cdot$ Ryota Kurayama $^{10}$. Shinsuke Matsumoto ${ }^{11}$. Yuhji Marui ${ }^{12}$. Shigeo Hara ${ }^{13}$. Shoichi Maruyama ${ }^{14} \cdot$ Ichiei Narita $^{15} \cdot$ Hirokazu Okada $^{16}$. Yoshifumi Ubara ${ }^{17}$ on behalf of Committee of Practical Guide for Kidney Biopsy 2019

1 Department of Nephrology, National Hospital Organization Chibahigashi National Hospital, 673 Nitona-cho, Chuo-ku, Chiba 260-8712, Japan

2 Division of Nephrology, Endocrinology, and Vascular Medicine, Tohoku University Hospital, Sendai, Japan

3 Department of Nephrology, Nara Medical University, Nara, Japan

4 Department of Pediatric Nephrology, Tokyo Women's Medical University, Tokyo, Japan

5 Department of Nephrology and Rheumatology, Aichi Medical University, Aichi, Japan

6 Department of Nephrology and Hypertension, Osaka City General Hospital, Osaka, Japan

7 Department of Nephrology, Mie University, Mie, Japan

8 Department of Nephrology and Rheumatology, National Center for Child Health and Development, Tokyo, Japan

9 Department of Pediatrics, Osaka Medical College, Osaka, Japan
10 Department of Pediatrics, Kosei General Hospital, Tokyo, Japan

11 Department of Pediatrics, Matsudo City General Hospital, Chiba, Japan

12 Department of Urology, St. Marianna University School of Medicine, Kawasaki, Japan

13 Department of Pathology, Kobe City Medical Center General Hospital, Kobe, Japan

14 Department of Nephrology, Nagoya University, Nagoya, Japan

15 Division of Clinical Nephrology and Rheumatology, Niigata University, Niigata, Japan

16 Department of Nephrology, Saitama Medical University, Saitama, Japan

17 Department of Nephrology, Toranomon Hospital, Tokyo, Japan 